\title{
Daytime sleepiness predicts inflammation and ambulatory blood pressure in sleep apnoea
}

\section{Victoria M. Pak (1) ${ }^{1}$, Brittany Butts ${ }^{1}$, Vicki Hertzberg (1) ${ }^{1}$, Nancy Collop², Arshed A. Quyyumi ${ }^{2}$, John Cox ${ }^{1}$, Ann Rogers ${ }^{1}$ and Sandra B. Dunbar ${ }^{1}$}

Affiliations: ${ }^{1}$ Emory Nell Hodgson Woodruff School of Nursing, Atlanta, GA, USA. ${ }^{2}$ Emory School of Medicine, Atlanta, GA, USA.

Correspondence: Victoria M. Pak, Emory Nell Hodgson Woodruff School of Nursing, 1520 Clifton Rd Atlanta, GA 30322, USA. E-mail: victoria.m.pak@emory.edu

\section{ABSTRACT}

Introduction: Sleepiness in obstructive sleep apnoea is associated with cardiovascular risk; however, the biological mechanisms are not known. This study explored whether those with subjective sleepiness have increased plasma tumour necrosis factor-related protein 1 (C1qTNF1), a novel adipose-derived hormone (adipokine), and 24-h ambulatory blood pressure (ABP) compared to those without sleepiness in newly diagnosed, treatment-naïve participants with obstructive sleep apnoea.

Methods: Overall, 94 participants were included in the analysis. Participants completed the Epworth Sleepiness Scale (ESS), 24-h ABP was monitored, and plasma C1qTNF1 was measured. Sleepy participants were defined as ESS $\geqslant 10$ and nonsleepy as ESS $<10$. Multiple linear regression was used to explore differences in C1qTNF1, and 24-h mean arterial pressure (MAP) between sleepy and nonsleepy participants, adjusting for age, sex, body mass index, apnoea-hypopnoea index, and smoking status.

Results: C1qTNF1 was significantly higher in sleepy participants $(n=57)$ compared to nonsleepy participants $(\mathrm{n}=37)(\beta=0.41 \mathrm{NPX}, 95 \%$ CI $0.02,0.80 ; \mathrm{p}=0.04)$. The 24 -h MAP was significantly higher in sleepy participants compared to nonsleepy participants $(\beta=4.06 \mathrm{mmHg}, 95 \%$ CI $0.36,7.77$; $\mathrm{p}=0.03$ )

Conclusions: Our findings show that sleepiness is associated with inflammation and higher 24-h MAP in sleep apnoea.

@ERSpublications

Excessive sleepiness experienced by treatment-naïve patients with obstructive sleep apnoea is associated with inflammation, higher daily systolic ambulatory blood pressure and higher $24 \mathrm{~h}$ mean arterial pressure https://bit.ly/3goeqGD

Cite this article as: Pak VM, Butts B, Hertzberg V, et al. Daytime sleepiness predicts inflammation and ambulatory blood pressure in sleep apnoea. ERJ Open Res 2020; 6: 00310-2019 [https://doi.org/ 10.1183/23120541.00310-2019].

This article has supplementary material available from openres.ersjournals.com.

Received: 8 Nov 2019 | Accepted after revision: 19 May 2020

Copyright $\odot$ ERS 2020. This article is open access and distributed under the terms of the Creative Commons Attribution Non-Commercial Licence 4.0. 


\section{Introduction}

Obstructive sleep apnoea (OSA) is a leading public health problem with an estimated prevalence rate of $34 \%$ in men and $17 \%$ in women in the United States [1]. Approximately $16-23 \%$ of those with OSA experience excessive daytime sleepiness (EDS) [2]. EDS is associated with a higher risk of cardiovascular disease (CVD), stroke and total as well as cardiovascular-specific mortality [3-7], both in those with sleep apnoea and the general population. A greater understanding of the biological basis of sleepiness symptoms in OSA and their relationship to cardiovascular risk is essential for developing interventions to reduce or minimise sleepiness symptoms and improve cardiovascular outcomes.

It is possible that the increased risk of sleepiness in OSA may be attributed to increased oxidative stress and inflammation caused by frequent and cyclic reductions in oxygen and rapid reoxygenation during sleep (i.e. cyclical intermittent hypoxia) [8]. In addition to hypoxia, excessive sleepiness in OSA is also likely to be caused by increased sleep fragmentation due to recurrent arousal $[9,10]$ and poor quality of sleep, as demonstrated by longer sleep duration and increased time spent in N1 (NREM1) as compared to slow-wave sleep $[11,12]$. We have previously explored genetic and metabolomic differences between sleepy and nonsleepy subjects $[13,14]$, suggesting that inflammation and oxidative stress are possible pathways for the observed increased risk of CVD. Growing evidence supports OSA as an independent risk factor for CVD, including hypertension, stroke, heart failure, and atrial fibrillation $[15,16]$. The process of intermittent hypoxia itself leads to increased blood pressure (BP), as sympathetic tone is activated in response to upper airway collapse during sleep in order to increase intrathoracic pressure and respiratory effort $[17,18]$. The increase in sympathetic tone and BP becomes consistent during both sleep and waking periods [17]. Additionally, hypoxia-induced reactive oxygen species triggers upregulation of transcription factor nuclear factor (NF)- $\mathrm{B}$, leading to the production of inflammatory biomarkers, such as intercellular adhesion molecule (ICAM)-1, tumour necrosis factor (TNF)- $\alpha$, and interleukin (IL)-6, which play a role in the atherosclerotic disease process $[8,19]$. Although several studies have explored sleepiness and $24 \mathrm{~h}$ ambulatory blood pressure [20-23], no previous study has explored inflammatory biomarker levels, sleepiness, and 24-h ambulatory BP (ABP) in those with newly diagnosed untreated sleep apnoea.

Complement C1q TNF-related protein 1 (C1qTNF1) is a novel adipose-derived hormone (adipokine) that is involved in inflammation and implicated in the pathogenesis of atherosclerosis and coronary artery disease [24-26]. This novel hormone is further pertinent as C1q remains a vital component of the classical complement pathway, which plays a main role in the clearance of foreign particles and is also involved in several other immunological processes such as the maintenance of immune tolerance, phagocytosis of bacteria, neutralisation of retroviruses, cell adhesion, and the modulation of cells [27, 28]. CIqTNF1 and TNF are positively correlated [29-31]. In addition, CIqTNF1 is increased in those with hypertension compared to healthy controls [32,33]. The purpose of this study is to explore the associations of C1qTNF1, a marker of inflammation, 24-h BP, and sleepiness in subjects with sleep apnoea. We hypothesised that mechanisms of sleepiness and CVD share common molecular pathways, thus subjects exhibiting sleepiness will have higher levels of inflammation and BP than subjects with sleep apnoea without sleepiness.

\section{Materials and methods}

\section{Study subjects and sleep apnoea diagnosis}

This was an exploratory study consisting of a sample of 94 participants from the Emory Mechanisms of Sleepiness Symptoms Study with available data conducted in 2017. All participants underwent an overnight sleep study at the laboratory or at home for the diagnosis of sleep apnoea, and subjects with an apnoea-hypopnoea index $(\mathrm{AHI}) \geqslant 5$ were recruited from the Emory Sleep Center. Participants were excluded from the study if they were under 22 years of age, night-shift workers, had presence of chronic obstructive pulmonary disease, liver cirrhosis, thyroid dysfunction, rheumatoid arthritis, chronic renal failure and/or psychiatric disorders, or a sleep disorder in addition to OSA based on polysomnography, such as restless legs syndrome or periodic limb movement disorder.

At home, sleep apnoea testing was conducted with the Embla MPR device. Oxyhaemoglobin saturation by pulse oximetry, thoracic and abdominal respiratory effort, nasal airflow by nasal cannula, and body position were monitored. In-laboratory recordings included electroencephalography, electrooculogram, ECG, chin and limb electromyelogram, chest and abdominal piezo belts, finger oximeter, and nasal pressure transducers.

The American Academy of Sleep Medicine acceptable scoring method was used to score the studies [34]. Trained sleep technicians scored polysomnograms. An apnoea was scored when there was $>10 \mathrm{~s}$ of airflow cessation, and a hypopnoea was defined by the presence of $4 \%$ desaturation during sleep associated with a reduction in nasal cannula or effort for $>10$ seconds. Intraindividual agreement between home and in-lab sleep study is high [35]. Fasting morning blood plasma samples were collected after the sleep study 
confirmed a diagnosis of sleep apnoea. The protocol was approved by the Emory Institutional Review Board and all patients provided informed consent.

\section{4-h ambulatory blood pressure monitoring}

All participating patients underwent 24-h ABP monitoring (Spacelabs, Medical Inc., Redmon, WA, USA) after being newly diagnosed with sleep apnoea and not yet treated on continuous positive airway pressure. A trained coordinator fitted an appropriately sized cuff on the patient's nondominant arm, which was worn for the subsequent $24 \mathrm{~h}$ during normal daily activities. The monitors were programmed to record $\mathrm{BP}$ every $20 \mathrm{~min}$ during the daytime (07:00-23:00 h) and every $30 \mathrm{~min}$ during the night-time period (23:00-07:00 h). Mean $24 \mathrm{~h}$ MAP was the primary cardiovascular outcome of interest. Supplemental analyses on daytime, night-time, 24-h systolic BP, and 24-h diastolic BP were also explored for the analysis.

\section{Plasma protein profiling using Olink multiplex panel}

C1qTNF1 was explored as a post hoc outcome exploratory measure and quantified using Olink multiplex proximity extension assay (PEA) panels (Olink Proteomics, Uppsala, Sweden) according to the manufacturer's instructions as described previously [36]. The data are presented as normalised protein expression (NPX) values, Olink Proteomics' arbitrary unit on $\log 2$ scale. In this study, the Olink Cardiometabolic panel was used to measure the C1qTNF1 protein in the plasma samples. The basis of PEA is a dual-recognition immunoassay, where two matched antibodies labelled with unique DNA oligonucleotides simultaneously bind to a target protein in solution. This brings the two antibodies into proximity, allowing their DNA oligonucleotides to hybridise, serving as a template for a DNA polymerase-dependent extension step. This creates a double-stranded DNA "barcode" that is unique for the specific antigen and is quantitatively proportional to the initial concentration of target protein. The hybridisation and extension are immediately followed by PCR amplification, and the amplicon is then finally quantified by microfluidic quantitative PCR using Fluidigm BioMark HD system (Fluidigm Corporation, South San Francisco, CA, USA).

\section{Sleepiness measurement}

The Epworth Sleepiness Scale (ESS), a standardised self-report instrument that assesses tendency to doze [37], was used to measure sleepiness. Participants were asked to rate their chance of dozing during eight common situations. The responses are based on a Likert-type scale ranging from 0 to 3, with 0 indicating no chance of dozing and 3 indicating a high chance of dozing. The sum of these responses determines the total ESS score, with higher scores indicating greater sleepiness [37]. Subjects were categorised as sleepy if they had an ESS score $\geqslant 10$ and nonsleepy if they had an ESS score $<10$ based on previous studies [38, 39].

\section{Statistical analysis}

Patient demographics, clinical characteristics and biomarker values are expressed as mean \pm SD. Categorical variables are described as frequencies and percentages. Univariate analyses were first performed to compare differences between patients with and without sleepiness, with a two-sample t-test or Wilcoxon rank-sum test used for continuous variables, and a Chi-squared or Fisher's exact test used for categorical variables. We applied multiple linear regression in which each dependent variable (24-h MAP, average

\begin{tabular}{|c|c|c|c|c|}
\hline Characteristic & Total $n=94$ & No EDS $n=37$ & EDS $n=57$ & p-value \\
\hline Age years & $50.5 \pm 12.8$ & $51.5 \pm 13.8$ & $49.8 \pm 12.2$ & 0.52 \\
\hline Sex male & $47(52.8 \%)$ & $20(54.1 \%)$ & $27(51.9 \%)$ & 0.84 \\
\hline Body mass index $\mathrm{kg} \cdot \mathrm{m}^{-2}$ & $35.9 \pm 9.4$ & $35.2 \pm 6.9$ & $36.3 \pm 10.7$ & 0.54 \\
\hline Apnoea-hypopnoea index & $32.0 \pm 26.1$ & $27.9 \pm 21.7$ & $34.6 \pm 28.5$ & 0.20 \\
\hline Current smoker & $13(13.8 \%)$ & $4(10.8 \%)$ & $9(15.8 \%)$ & 0.55 \\
\hline Epworth Sleepiness Scale (0-24) & $11.0 \pm 5.3$ & $5.4 \pm 2.3$ & $14.6 \pm 3.2$ & $<0.01$ \\
\hline Overnight & 33 (35.1\%) & $11(29.7 \%)$ & $22(38.6 \%)$ & 0.51 \\
\hline Home & $61(64.9 \%)$ & $26(70.3 \%)$ & $35(61.4 \%)$ & 0.51 \\
\hline
\end{tabular}


daytime systolic BP, average daytime diastolic BP, average night-time systolic BP, average night-time diastolic $\mathrm{BP}$, and the biomarker value (C1qTNF1)) was regressed separately against the binary sleepiness factor, adjusting for age, sex, BMI, AHI, and smoking status. Version 3.4 .4 of $\mathrm{R}$ for Windows ( Foundation for Statistical Computing, Vienna, Austria) was used for the analysis. Significance was set at a two-tailed p-value of 0.05 .

\section{Results}

Summary statistics of patient demographics are presented in table 1 and characteristics of study population variables specific to the cardiovascular outcomes, comorbidities, and C1qTNF1 are in table 2. The mean \pm SD age was $49.8 \pm 12.2$ in 57 sleepy subjects and $51.5 \pm 13.8$ in 37 nonsleepy subjects. There were 47 $(52.8 \%)$ males in the cohort. There were 94 subjects with available biomarker and ABP data. Overall, 13 were evaluated in the sleep laboratory and 81 were evaluated on home sleep study. There were no significant differences between overnight sleep study and home study between sleepy and nonsleepy subjects, as expected. There were also no differences in dipping status between sleepy and nonsleepy subjects. Overall, the mean of the ESS scores was $11.0 \pm 5.3$ with $60.6 \%$ of the subjects $(n=57)$ categorised as having EDS. Other demographics, including BMI, AHI, smoking status, and history of stroke, myocardial infarction, hypertension, and heart failure were all comparable between the two groups (see table 1). Our primary analysis was focused on whether subjects with sleepiness had higher MAP $24 \mathrm{~h}$ measures adjusting for the aforementioned covariates. Subjects with sleepiness had higher adjusted MAP $24 \mathrm{~h}(\beta$ $4.06 \mathrm{mmHg}, 95 \% \mathrm{CI} 0.36,7.77 ; \mathrm{p}=0.03$ ), and higher univariate MAP $24 \mathrm{~h}$ ( $\beta 4.61 \mathrm{mmHg}, 95 \%$ CI 0.741 , 8.48; $\mathrm{p}=0.02)$. Compared to those who were nonsleepy $(\mathrm{n}=37)$, those who were sleepy $(\mathrm{n}=57)$ had significantly higher adjusted C1qTNF levels ( $\beta=0.41 \mathrm{NPX}, 95 \%$ CI 0.02, 0.80; $\mathrm{p}=0.04$ ) (figure 1).

See table 3 for adjusted regression models and supplemental table 1 for unadjusted models. We also re-ran the analyses without a major outlier to ensure that the observed effect of sleepiness on C1qTNF1 levels on $24 \mathrm{~h}$ MAP was not driven by outliers, and the main results remained stable and did not change significantly.

\section{Discussion}

We have identified associations worth exploring in larger studies between sleepiness, C1qTNF1 and $24 \mathrm{~h}$ MAP in newly diagnosed sleep apnoeic subjects. Compared to those who were nonsleepy, those with

\begin{tabular}{|c|c|c|c|c|}
\hline Characteristic & Total $n=94$ & No EDS $n=37$ & EDS $n=57$ & p-value \\
\hline \multicolumn{5}{|l|}{ 24-h ambulatory BP mmHg ${ }^{\#}$} \\
\hline MAP $24 \mathrm{~h}$ & $91.3 \pm 8.5$ & $88.8 \pm 8.1$ & $92.8 \pm 8.4$ & 0.03 \\
\hline SBP wake (average daily systolic) & $128.5 \pm 14.5$ & $125.4 \pm 14.6$ & $130.3 \pm 14.2$ & 0.13 \\
\hline DBP wake (average daily diastolic) & $77.8 \pm 7.9$ & $76.4 \pm 7.3$ & $78.7 \pm 8.2$ & 0.16 \\
\hline MAP wake & $93.9 \pm 8.5$ & $91.8 \pm 8.1$ & $95.3 \pm 8.5$ & 0.06 \\
\hline SBP sleep (average nightly systolic) & $120.0 \pm 17.3$ & $116.0 \pm 17.7$ & $122.4 \pm 16.8$ & 0.11 \\
\hline DBP sleep (average nightly diastolic) & $70.1 \pm 9.4$ & $67.3 \pm 9.1$ & $71.7 \pm 9.2$ & 0.04 \\
\hline MAP sleep & $86.1 \pm 10.1$ & $83.0 \pm 10.5$ & $88.0 \pm 9.5$ & 0.03 \\
\hline SBP $24 \mathrm{~h}$ & $125.6 \pm 14.8$ & $122.5 \pm 14.8$ & $127.5 \pm 14.5$ & 0.13 \\
\hline DBP $24 \mathrm{~h}$ & $75.2 \pm 7.8$ & $73.4 \pm 7.0$ & $76.4 \pm 8.1$ & 0.07 \\
\hline Dipper systolic & $6.4 \pm 7.7$ & $6.7 \pm 8.3$ & $6.3 \pm 7.4$ & 0.83 \\
\hline Dipper diastolic & $9.6 \pm 8.1$ & $10.7 \pm 8.8$ & $8.9 \pm 7.8$ & 0.34 \\
\hline Dipper MAP & $8.1 \pm 7.4$ & $9.2 \pm 8.1$ & $7.5 \pm 6.9$ & 0.32 \\
\hline Stroke (yes) & $5(5.3 \%)$ & $1(2.7 \%)$ & $4(7.0 \%)$ & 0.65 \\
\hline Myocardial infarction (yes) & $2(2.1 \%)$ & $1(2.7 \%)$ & $1(1.8 \%)$ & 1.00 \\
\hline Hypertension (yes) & $40(43.5 \%)$ & $16(43.2 \%)$ & $24(43.6 \%)$ & 1.00 \\
\hline Heart failure (yes) & $7(7.4 \%)$ & $2(5.4 \%)$ & $5(8.8 \%)$ & 0.70 \\
\hline C1qTNF1 NPX & $6.0 \pm 0.9$ & $5.7 \pm 0.7$ & $6.1 \pm 1.0$ & 0.02 \\
\hline
\end{tabular}

Data are presented as mean \pm SD; categorical variables are presented as $\mathrm{n}(\%)$. C1qTNF1: tumour necrosis factor-related protein 1; EDS: excessive daytime sleepiness; BP: blood pressure; SBP: systolic blood pressure; DBP: diastolic blood pressure; MAP: mean arterial pressure; NPX: normalised protein expression. \#: there were seven individuals missing 24-h BP data. Some reported characteristics are missing values which were excluded from the total summary data. A two-sample t-test or Wilcoxon rank-sum test was used for continuous variables, and a Chi-squared or Fisher's exact test was used for categorical variables. 
FIGURE 1 Differences in tumour necrosis factor-related protein 1 (C1qTNF1) concentration between subjects with sleepiness versus those without. ESS: Epworth Sleepiness Score; NPX: normalised protein expression.

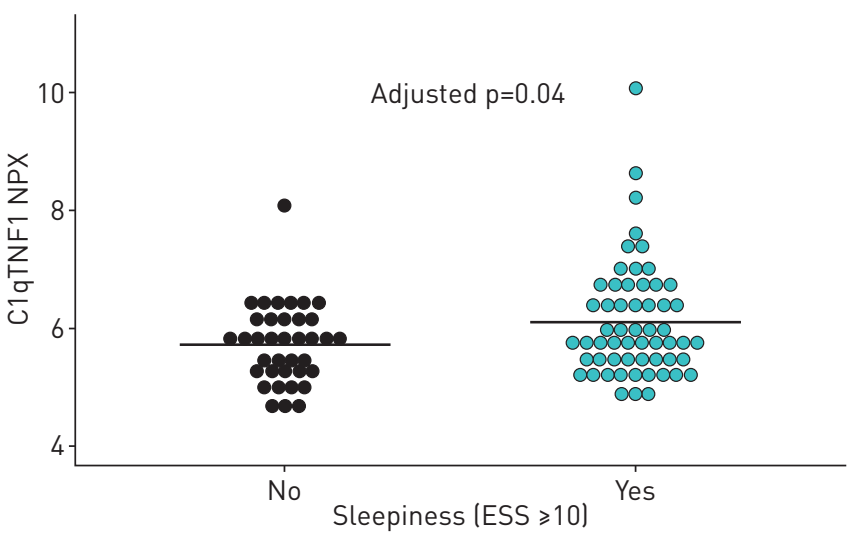

sleepiness had significantly higher C1qTNF1 levels and 24-h MAP BP. Limitations of our study include possible measurement inconsistency in the ESS; however, as subjects are asked to recall their propensity to sleep over the past few weeks rather than just at the time of testing, the ESS is built to address this bias. Results are expected to be generalisable to newly diagnosed sleep apnoeic subjects with moderate sleep apnoea. Although relationships between 24-h MAP and daytime sleepiness via the ESS have been noted $[23,40]$, we are the first to explore C1qTNF1 which is linked to low-grade inflammation to explain the relationship. Our results are congruent with a previous study examining the relationship between subjective sleepiness according to ESS and hypertension in OSA patients; OSA patients with excessive sleepiness had increased odds of hypertension by $23 \%$ as compared to OSA patients without EDS [41]. Similarly, a study investigating objective daytime sleepiness as assessed by the multiple sleep latency test and hypertension in OSA patients, found a significant positive association between sleepiness and hypertension [42].

C1qTNF1 is a complement the C1q/TNF-related protein family adipokine that is expressed mainly in adipose stromal vascular cells. C1qTNF1 has been linked to low-grade chronic inflammation in adipose tissue [43], and has been shown to upregulate inflammatory genes, such as IL6 and ICAM1, in vascular smooth muscle [44], suggesting that C1qTNF1 may be a pro-atherogenic biomarker. Under conditions of disturbed flow, C1qTNF1 promotes endothelium-leukocyte interactions and inflammatory responses in vascular cells [25], which increases endothelial permeability and further contributes to atherogenesis. C1qTNF1 is increased in patients with coronary artery disease (CAD), and has been found in the serum, atherosclerotic plaques, and peripheral blood mononucleocytes of people with CAD [24]. A longitudinal study of 539 white patients referred to elective coronary angiography for the evaluation of established or suspected stable CAD who were followed for major adverse cardiovascular events (MACE). This was

TABLE 3 Results of the multiple regression analyses of C1qTNF1 and ambulatory blood pressure measures as a function of sleepiness (yes versus no) and adjusted covariates

\begin{tabular}{|c|c|c|c|}
\hline Dependent variables & Estimate & SE & $\mathrm{p}$-value \\
\hline C1qTNF1 NPX & 0.41 & 0.20 & 0.04 \\
\hline SBP wake $\mathrm{mmHg}$ & 6.28 & 3.13 & 0.05 \\
\hline DBP wake $\mathrm{mmHg}$ & 2.37 & 1.81 & 0.19 \\
\hline SBP sleep $\mathrm{mmHg}$ & 5.96 & 3.81 & 0.12 \\
\hline DBP sleep $\mathrm{mmHg}$ & 3.44 & 1.91 & 0.08 \\
\hline SBP $24 \mathrm{~h} \mathrm{mmHg}$ & 6.11 & 3.20 & 0.06 \\
\hline DBP $24 \mathrm{~h} \mathrm{mmHg}$ & 2.81 & 1.68 & 0.10 \\
\hline Dipper systolic mmHg & 0.19 & 1.77 & 0.92 \\
\hline Dipper diastolic $\mathrm{mmHg}$ & -1.02 & 1.85 & 0.59 \\
\hline Dipper MAP mmHg & -0.79 & 1.70 & 0.64 \\
\hline MAP $24 \mathrm{~h} \mathrm{mmHg}$ & 4.06 & 1.86 & 0.03 \\
\hline MAP wake $\mathrm{mmHg}$ & 3.78 & 1.93 & 0.05 \\
\hline MAP sleep $\mathrm{mmHg}$ & 4.56 & 2.17 & 0.04 \\
\hline
\end{tabular}

C1qTNF1: tumour necrosis factor-related protein 1; SBP: systolic blood pressure; DBP: diastolic blood pressure; MAP: mean arterial pressure; NPX: normalised protein expression. 
defined as a composite of cardiovascular death, nonfatal myocardial infarction, and nonfatal stroke, for an average of 5.9 years found that increased serum C1qTNF1 was associated with an increased risk for future MACE [45].

Taken together, the higher levels of the inflammatory protein C1qTNF1 in people with OSA and EDS may indicate not just a higher inflammatory state but also increased risk of CVD and adverse outcomes. C1qTNF1 has also been shown to positively regulate BP via increased vasoconstriction and stimulation of aldosterone production [33], resulting in both increased peripheral vascular resistance and total blood volume. C1qTNF1 acts as a long-term regulator of BP and is a key effector of glucocorticoid-mediated BP regulation, as glucocorticoid administration in murine models increases C1qTNF1 production [46].

Considering the link between sleep-disordered breathing and cortisol dysregulation [47, 48], it is possible that higher levels of C1qTNF1 may link the proinflammatory state related to cyclical intermittent hypoxia to the higher BP in people with OSA and EDS in this hypothesis-generating study and requires further exploration. The exploration of other inflammatory biomarkers and their associations with sleepiness and cardiovascular outcomes will be important for future studies aimed at targeted treatments. Further work examining the role of inflammatory proteins in BP regulation in those with OSA and EDS is needed.

Conflict of interest: None declared.

Support statement: This work was supported by an R00NR01465-03 NIH Pathway to Independence award (V.M. Pak). Funding information for this article has been deposited with the Crossref Funder Registry.

\section{References}

1 Peppard PE, Young T, Barnet JH, et al. Increased prevalence of sleep-disordered breathing in adults. Am J Epidemiol 2013; 177: 1006-1014.

2 Franklin KA, Lindberg E. Obstructive sleep apnea is a common disorder in the population-a review on the epidemiology of sleep apnea. J Thorac Dis 2015; 7: 1311-1322.

3 Newman AB, Spiekerman CF, Enright P, et al. Daytime sleepiness predicts mortality and cardiovascular disease in older adults. The cardiovascular health study research group. J Am Geriatr Soc 2000; 48: 115-123.

4 Boden-Albala B, Roberts ET, Bazil C, et al. Daytime sleepiness and risk of stroke and vascular disease: findings from the Northern Manhattan Study (NOMAS). Circ Cardiovasc Qual Outcomes 2012; 5: 500-507.

5 Lee $\mathrm{CH}, \mathrm{Ng} \mathrm{WY}$, Hau W, et al. Excessive daytime sleepiness is associated with longer culprit lesion and adverse outcomes in patients with coronary artery disease. J Clin Sleep Med 2013; 9: 1267-1272.

6 Empana JP, Dauvilliers Y, Dartigues JF, et al. Excessive daytime sleepiness is an independent risk indicator for cardiovascular mortality in community-dwelling elderly: the three city study. Stroke 2009; 40: 1219-1224.

7 Qureshi AI, Giles WH, Croft JB, et al. Habitual sleep patterns and risk for stroke and coronary heart disease: a 10-year follow-up from NHANES I. Neurology 1997; 48: 904-911.

8 Pak VM, Grandner MA, Pack AI. Circulating adhesion molecules in sleep apnea and cardiovascular disease. Sleep Med Rev 2014; 18: 25-34.

9 Bennett LS, Langford BA, Stradling JR, et al. Sleep fragmentation indices as predictors of daytime sleepiness and nCPAP response in obstructive sleep apnea. Am J Respir Crit Care Med 1998; 158: 778-786.

10 Seneviratne U, Puvanendran K. Excessive daytime sleepiness in obstructive sleep apnea: prevalence, severity, and predictors. Sleep Med 2004; 5: 339-343.

11 Sun Y, Ning Y, Huang L, et al. Polysomnographic characteristics of daytime sleepiness in obstructive sleep apnea syndrome. Sleep Breath 2012; 16: 375-381.

12 Roure N, Gomez S, Mediano O, et al. Daytime sleepiness and polysomnography in obstructive sleep apnea patients. Sleep Med 2008; 9: 727-731.

13 Pak VM, Mazzotti DR, Keenan BT, et al. Candidate gene analysis in the Sao Paulo epidemiologic sleep study (EPISONO) shows an association of variant in PDE4D and sleepiness. Sleep Med 2018; 47: 106-112.

14 Pak VM, Dai F, Keenan BT, et al. Lower plasma choline levels are associated with sleepiness symptoms. Sleep Med 2018; 44: 89-96.

15 Tietjens JR, Claman D, Kezirian EJ, et al. Obstructive sleep apnea in cardiovascular disease: a review of the literature and proposed multidisciplinary clinical management strategy. J Am Heart Assoc 2019; 8: e010440.

16 Monahan K, Redline S. Role of obstructive sleep apnea in cardiovascular disease. Curr Opin Cardiol 2011; 26: $541-547$.

17 Somers VK, Dyken ME, Clary MP, et al. Sympathetic neural mechanisms in obstructive sleep apnea. J Clin Invest 1995; 96: 1897-1904.

18 Xie A, Skatrud JB, Puleo DS, et al. Exposure to hypoxia produces long-lasting sympathetic activation in humans. J Appl Physiol 2001; 91: 1555-1562.

19 Bauters F, Rietzschel ER, Hertegonne KB, et al. The link between obstructive sleep apnea and cardiovascular disease. Curr Atheroscler Rep 2016; 18: 1.

20 Gus M, e Silva DN, Fernandes J, et al. Epworth's sleepiness scale in outpatients with different values of arterial blood pressure. Arq Bras Cardiol 2002; 78: 21-24.

21 Hui DS, To KW, Ko FW, et al. Nasal CPAP reduces systemic blood pressure in patients with obstructive sleep apnoea and mild sleepiness. Thorax 2006; 61: 1083-1090.

22 Goldstein IB, Ancoli-Israel S, Shapiro D. Relationship between daytime sleepiness and blood pressure in healthy older adults. Am J Hypertens 2004; 17: 787-792.

23 Lloberes P, Lozano L, Sampol G, et al. Obstructive sleep apnoea and 24-h blood pressure in patients with resistant hypertension. J Sleep Res 2010; 19: 597-602. 
$24 \mathrm{Lu} \mathrm{L}$, Zhang RY, Wang XQ, et al. C1q/TNF-related protein-1: an adipokine marking and promoting atherosclerosis. Eur Heart J 2016; 37: 1762-1771.

25 Liu ZH, Li C, Chen JW, et al. C1q/TNF-related protein 1 promotes endothelial barrier dysfunction under disturbed flow. Biochem Biophys Res Commun 2017; 490: 580-586.

26 Tang JN, Shen DL, Liu CL, et al. Plasma levels of C1q/TNF-related protein 1 and interleukin 6 in patients with acute coronary syndrome or stable angina pectoris. Am J Med Sci 2015; 349: 130-136.

27 Kishore U, Gaboriaud C, Waters P, et al. C1q and tumor necrosis factor superfamily: modularity and versatility. Trends Immunol 2004; 25: 551-561.

28 Schäffler A, Buechler C. CTRP family: linking immunity to metabolism. Trends Endocrinol Metab 2012; 23 194-204

29 Xin Y, Lyu X, Wang C, et al. Elevated circulating levels of CTRP1, a novel adipokine, in diabetic patients. Endocr J 2014; 61: 841-847.

30 Shen L, Wang S, Ling Y, et al. Association of C1q/TNF-related protein-1 (CTRP1) serum levels with coronary artery disease. J Int Med Res 2019; 47: 2571-2579.

31 Kim K-Y, Kim HY, Kim JH, et al. Tumor necrosis factor- $\alpha$ and interleukin-1 $\beta$ increases CTRP1 expression in adipose tissue. FEBS Lett 2006; 580: 3953-3960.

32 Jeon JH, Kim KY, Kim JH, et al. A novel adipokine CTRP1 stimulates aldosterone production. FASEB J 2008; 22 : $1502-1511$

33 Han S, Yang Y. A novel blood pressure modulator C1q/TNF- $\alpha$-related protein 1 (CTRP1). BMB Rep 2018; 51 611-612.

34 Iber C A-IS, Chesson A, Quan S. The AASM Manual for the Scoring of Sleep and Associated Events: Rules, Terminology and Technical Specifications. 1st Edn. American Academy of Sleep Medicine, 2007.

35 Harris L, Swinson K. Home sleep testing for diagnosing obstructive sleep apnea. Am Fam Physician 2017; 96 Online.

36 Assarsson E, Lundberg M, Holmquist G, et al. Homogenous 96-plex PEA immunoassay exhibiting high sensitivity, specificity, and excellent scalability. PLoS One 2014; 9: e95192.

37 Johns MW. A new method for measuring daytime sleepiness: the Epworth Sleepiness Scale. Sleep 1991; 14 540-545.

38 Yüksel P, Glantz H, Eulenburg C, et al. Effect of positive airway pressure on cardiovascular outcomes in coronary artery disease patients with nonsleepy obstructive sleep apnea. The RICCADSA randomized controlled trial Am J Respir Crit Care Med 2016; 194: 613-620.

39 Hogl B, Saletu M, Brandauer E, et al. Modafinil for the treatment of daytime sleepiness in Parkinson's disease: a double-blind, randomized, crossover, placebo-controlled polygraphic trial. Sleep 2002; 25: 905-909.

40 Shapiro D, Ancoli-Israel S, Goldstein IB. Relationship between daytime sleepiness and blood pressure in healthy older adults. Am J Hypertens 2004; 17: 787-792.

$41 \mathrm{Du}$ LN, Ren R, Tan L, et al. [Association between daytime sleepiness and hypertension among patients with obstructive sleep apnea-hypopnea syndrome]. Zhonghua Yi Xue Za Zhi 2016; 96: 3370-3374.

42 Ren $\mathrm{R}$, Li Y, Zhang J, et al. Obstructive sleep apnea with objective daytime sleepiness is associated with hypertension. Hypertension 2016; 68: 1264-1270.

43 Kim KY, Kim HY, Kim JH, et al. Tumor necrosis factor- $\alpha$ and interleukin-1beta increases CTRP1 expression in adipose tissue. FEBS Lett 2006; 580: 3953-3960.

44 Kim D, Park SY. C1q and TNF related protein 1 regulates expression of inflammatory genes in vascular smooth muscle cells. Genes Genomics 2019; 41: 397-406.

45 Muendlein A, Leiherer A, Saely C, et al. The novel adipokine CTRP1 is significantly associated with the incidence of major adverse cardiovascular events. Atherosclerosis 2019; 286: 1-6.

46 Han S, Jeong AL, Lee S, et al. C1q/TNF- $\alpha$-related protein 1 (CTRP1) maintains blood pressure under dehydration conditions. Circ Res 2018; 123: e5-e19.

47 Ghiciuc CM, Dima-Cozma LC, Bercea RM, et al. Imbalance in the diurnal salivary testosterone/cortisol ratio in men with severe obstructive sleep apnea: an observational study. Braz J Otorhinolaryngol 2016; 82: 529-535.

48 Mehra R, Wang L, Andrews N, et al. Dissociation of objective and subjective daytime sleepiness and biomarkers of systemic inflammation in sleep-disordered breathing and systolic heart failure. J Clin Sleep Med 2017; 13: $1411-1422$. 